\title{
Valoración del trabajo colaborativo y rendimiento académico en el proceso de enseñanza de un curso de investigación en estudiantes de medicina
}

\section{Valuation of collaborative work and academic performance in the teaching process of a research course in medical students}

\author{
Juan P. Matzumura-Kasano ${ }^{1,2, a}$, Hugo Gutiérrez-Crespo ${ }^{1,2, b}$, César Pastor-Garcia ${ }^{2, c}$, Raúl A. Ruiz-Arias ${ }^{2,3, d}$ \\ ${ }^{1}$ Sociedad Peruana de Obstetricia y Ginecología. Lima, Perú. \\ ${ }^{2}$ Facultad de Medicina, Universidad Nacional Mayor de San Marcos. Lima, Perú. \\ ${ }^{3}$ Universidad San Ignacio de Loyola. Lima, Perú. \\ ${ }^{a}$ Médico ginecoobstetra, doctor en medicina. ORCID: http://orcid.org/0000-0003-0231-0187 \\ ${ }^{\mathrm{b}}$ Obstetra, magíster en docencia e investigación en salud, ORCID: http://orcid.org/0000-0003-1097-6990 \\ ${ }^{\circ}$ Médico cirujano, especialista en medicina interna, ORCID: http://orcid.org/0000-0001-8112-6120 \\ ${ }^{\mathrm{d}}$ Estadístico, ORCID: http://orcid.org/0000-0002-8877-6158
}

An Fac med. 2019;80(4):457-64 / DOI: https://doi.org/10.15381/anales.v80i4.17251

\begin{abstract}
Correspondencia:
Juan P. Matzumura Kasano

jmatzumura@yahoo.com
\end{abstract}

Recibido: 4 de noviembre 2019

Aceptado: 3 de diciembre 2019

Publicación en línea: 28 de diciembre 2019

Conflictos de interés: Los autores declaran no tener conflictos de interés.

Fuente de financiamiento: Autofinanciado

Citar como: Matzumura-Kasano JP, Gutiérrez-Crespo HF, Pastor-García CA, Ruiz-Arias RA. Valoración del trabajo colaborativo y rendimiento académico en el proceso de enseñanza de un curso de investigación en estudiantes de medicina. An Fac med. 2019;80(4):45764. DOI: https:/doi.org/10.15381/anales. v80i4.17251

\section{Resumen}

Introducción. El trabajo colaborativo incentiva el aprendizaje mediante la participación activa de los estudiantes, permitiendo desarrollar habilidades sociales y construir sus propios conocimientos. Objetivo. Analizar el trabajo colaborativo y su relación con el rendimiento académico en estudiantes de medicina de un curso de investigación. Métodos. Estudio transversal, en el que participaron 148 estudiantes de medicina humana matriculados en el curso de investigación, seleccionados mediante una muestra no probabilística. Se utilizó el cuestionario denominado análisis del trabajo cooperativo en educación superior que valora la importancia del trabajo colaborativo, de 49 preguntas distribuidas en siete dimensiones. Se utilizó una escala de Likert en un rango de 1 a 5, donde 1 se valora como total desacuerdo y 5 totalmente de acuerdo. Se establecieron tres grupos: grupo bajo hasta percentil 33; grupo medio, de percentil 34 a percentil 66; y grupo alto, de percentil 67 a más. Resultados. La edad promedio fue 22,4 años y $52,7 \%$ correspondieron al género femenino. En la dimensión "concepción del trabajo", 40,5\% de estudiantes pertenecieron al grupo medio; en la dimensión "planificación del trabajo de los grupos por el profesor", 41,9\% al grupo bajo; en "criterios para organizar grupos", 37,8\% al grupo bajo; en "funcionamiento interno", 43,2\% al grupo bajo; y en "eficacia del trabajo", $32,4 \%$ al grupo alto y $39,9 \%$ al grupo bajo. El promedio global de notas correspondió a 12,7. El rendimiento académico en estudiantes del grupo bajo y medio fue similar, y en las estudiantes de género femenino del grupo alto fue significativamente mayor $(p=0,049)$. Conclusiones. El grupo bajo predominó en las dimensiones "planificación del trabajo del profesor", "criterios para organizar grupos", "normas de funcionamiento", "funcionamiento interno" y "eficacia". 54,7\% de estudiantes correspondió al grupo alto y el promedio global de calificaciones fue 12,7. No existió relación entre las variables estudiadas.

Palabras clave: Aprendizaje Colaborativo; Rendimiento Académico; Investigación; Cursos; Facultades de Medicina (fuente: DeCS BIREME).

\section{Abstract}

Introduction. Collaborative work promotes learning through active participation of students, allowing the development of social abilities and the construction of their own knowledge. Objective. To analyze collaborative work and its relationship with academic performance in medical students in a research course. Methods. Cross-sectional study, in which 148 human medicine students enrolled in the research course participated, selected using a non-probabilistic sample. The questionnaire called analysis of cooperative work in higher education that assesses the importance of collaborative work, of 49 questions distributed in seven dimensions, was used. A Likert scale was used in a range of 1 to 5 , where 1 is valued as a total disagreement and 5 totally agree. Three groups were established: low group to 33 percentile; middle group, from 34th percentile to 66th percentile; and high group, from 67th percentile to more. Results. The average age was 22,4 years and $52,7 \%$ corresponded to the female gender. In the "conception of work" dimension, 40,5\% of students belonged to the middle group; in the dimension "planning the work of the groups by the teacher", $41,9 \%$ to the low group; in "criteria for organizing groups", $37,8 \%$ for the low group; in "internal operation", $43,2 \%$ to the low group; and in "work efficiency", $32,4 \%$ to the high group and $39,9 \%$ to the low group. The global average of grades corresponded to 12,7. The academic performance in students of the low and medium group was similar, and in the female students of the high group it was significantly higher $(p=0,049)$. Conclusions. The low group predominated in the dimensions "teacher's work planning", "criteria for organizing groups", "operating standards", "internal functioning" and "efficiency". $54,7 \%$ of students corresponded to the high group and the overall grade point average was 12,7 . There was no relationship between the variables studied.

Keywords: Collaborative Learning; Academic Performance; Research; Courses; Schools, Medical (source: MeSH NLM). 


\section{INTRODUCCIÓN}

El trabajo colaborativo, a diferencia del modelo tradicional, aporta algunos elementos didácticos y enriquecedores para el propio proceso de aprendizaje. Tal como sostienen Pico y Rodríguez, el trabajo colaborativo en el contexto del aula invita a docentes y estudiantes a caminar juntos, sumando esfuerzos, talentos y competencias; además, incentiva el aprender haciendo, el aprender interactuando y el aprender compartiendo ${ }^{(1)}$.

De acuerdo con las teorías actuales sobre el aprendizaje, la participación activa de estudiantes en el proceso de enseñanza-aprendizaje proporciona importantes beneficios frente a los métodos tradicionales, permitiendo desarrollar habilidades, actitudes, incrementar la retención de la información y construir relaciones con significado entre los conocimientos que posee al inicio y los que adquiere en el proceso de aprendizaje ${ }^{(2)}$.

Algunos estudiantes tienen dificultades para aprender, puesto que la mayoría de los profesores fueron formados según la educación tradicional, donde la práctica de la enseñanza significativa supone la concentración en la enseñanza de la teoría bajo el supuesto de que el conocimiento puede y debe ser trasmitido del profesor al estudiante. Asimismo, consideran que el proceso de aprendizaje es complejo e individualista y que, sumado a la corta disponibilidad de tiempo, no cumple con sus expectativas. Por ello, el resultado esperado es que el estudiante replique el contenido y la estructura del mundo en su pensamiento. Este enfoque muestra varios problemas: la enseñanza se centra en el saber qué y no en el cómo, y los nuevos conocimientos no ayudan al estudiante a alcanzar un objetivo que sea significativo ya que su aprendizaje se realiza de manera descontextualizada ${ }^{(3)}$.

Por otra parte, los instrumentos utilizados por los profesores como autoevaluación, seguimiento, tutorización de trabajo, memoria y exposición son válidos para valorar el trabajo grupal, pero insuficientes para determinar con exactitud la calificación individual. Calificar la dedicación individual con una nota grupal puede convertirse en un acto injusto e imprudente, debido a que se corre el riesgo de penalizar o premiar a unos estudiantes por la actuación de otros miembros del equipo, una calificación grupal que refleje de manera parcial la capacidad de los estudiantes. Para promover un verdadero aprendizaje colaborativo, los procesos de evaluación son cruciales y deben considerarse diversos aspectos: desarrollo de evaluación formativa y compartida, cuya finalidad principal sea la mejora de los procesos de aprendizaje del estudiante y el perfeccionamiento del profesor. Establecer un sistema de calificaciones que combine el rendimiento individual, grupal y proponer cierta diversidad de instrumentos de evaluación que abarquen actividades de autoevaluación y de evaluación compartida de grupos de trabajo ${ }^{(4)}$

Es importante resaltar que un equipo de aprendizaje colaborativo es más que un grupo de estudiantes que hacen algo entre todos; así, existe diferencia entre repartir el trabajo y la cooperación con otras personas para realizar un proyecto común. La cooperación supone plantear estructuras de aprendizaje donde hay un cambio sustancial en las formas de interacción entre estudiantes: compartir tareas entre todos contribuye al logro de una meta común ${ }^{(5,6)}$.

La experiencia en la docencia universitaria ha permitido evidenciar que aprender de forma colaborativa produce un incremento significativo en el rendimiento de los estudiantes, en comparación con otros grupos que siguen aprendiendo de forma individual. Es evidente que si hay una responsabilidad y un compromiso para la consecusión del logro del equipo el rendimiento académico se incrementará y con ello los estudiantes adquieren nuevos conocimientos (7). Por ello, se plantea como objetivo, analizar el trabajo colaborativo y su relación con el rendimiento académico en estudiantes de medicina de un curso de investigación, durante el año 2019.

\section{MÉTODOS}

\section{Diseño del estudio}

Se realizó un estudio observacional, correlacional de corte transversal.

\section{Población y muestra}

La población estuvo conformada por 166 estudiantes de medicina humana matriculados en el curso de metodología de la investigación médica de una universidad privada de Lima. Se incluyó a estudiantes que aceptaron participar de forma voluntaria y con asistencia $\geq 70 \%$. Fueron excluidos 18 estudiantes por presentar una inasistencia $\geq 30 \%$, por haberse retirado del curso y por no desean participar. El tipo de muestra correspondió a una muestra no probabilística por conveniencia, conformada por 148 estudiantes y la recolección de datos se realizó durante los meses de marzo a agosto del año 2019.

\section{Variables de estudio}

La recolección de datos se realizó a través de la aplicación de un cuestionario diseñado por García M et al. denominado: cuestionario de análisis del trabajo cooperativo en educación superior (ACOES) ${ }^{(5)}$; la medición utiliza una escala de Likert de carácter numérico en un rango de 1 a 5, donde 1 se valora como total desacuerdo y 5 totalmente de acuerdo. El cuestionario valora la importancia del trabajo colaborativo y esta conformado por 49 preguntas distribuidas en siete dimensiones: concepción del trabajo, utilidad del trabajo para su formación, planificación del trabajo por parte del profesor, criterios para organizar los grupos, normas, funcionamiento interno, y eficacia del trabajo grupal.

Para determinar las valoraciones finales se establecieron tres grupos: grupo bajo, valor mínimo, percentil 33 (151,173); grupo medio, percentil 34, percentil $66(174,193)$; y, grupo alto, percentil 67, valor máximo $(194,219)$. Este cuestionario tiene una fiabilidad total de 0,89 según alfa de cronbach.

Para el desarrollo de la investigación se consideró conveniente realizar actividades previas cortas según recomendaciones de estudios previos ${ }^{(7,8)}$, y de acuerdo a la siguiente descripción: 1) Socialización de competencias a lograr, revisión de sílabo y criterios de evaluación; 2) Análisis sobre las ventajas de utilizar trabajo colaborativo; 3) Conformación de equipos de trabajo por afinidad, con responsabilidad para el desarrollo de trabajos aplicativos, 
presentación de resúmenes, preparación de un proyecto de investigación; 4) Explicar la importancia de la participación activa del estudiante para producir conocimientos; y 5) Fomento de la creatividad e innovación y reconocimiento a los grupos que evidencian buen rendimiento.

El rendimiento académico fue medido mediante el promedio final de notas según escala vigesimal, de acuerdo a los siguientes componentes: a) calificaciones correspondientes a conocimientos; b) desarrollo de actividades aplicativas; c) evaluación actitudinal; y d) proyecto de investigación formativa.

\section{Análisis estadístico}

El procesamiento de datos se realizó mediante IBM SPSS Statistics for Windows, Version 22.0 (IBM Corp. Released $2013{ }^{\circledR}$ ), que apoyó en el cálculo de medias, proporciones, y tendencias. Asimismo, se analizaron las diferencias entre valoraciones sobre el trabajo en equipo y géneros, así como el promedio de notas y el trabajo colaborativo, mediante la prueba de $U$ de Mann-Whitney. Se consideró estadísticamente significativo un valor $p<0,05$.

\section{Aspectos éticos}

La investigación se realizó de acuerdo con las consideraciones éticas, las buenas prácticas en investigación, respetando la integridad y confidencialidad de los participantes.

\section{RESULTADOS}

Los datos generales permitieron establecer que 52,7\% (78 estudiantes) correspondieron al género femenino, con una edad promedio para el género femenino de 22,9 $\pm 6,3$ años $y$, para el género masculino de 21,8 \pm 4,2 años. La edad promedio global fue de $22,4 \pm 5,6$ años.

Los resultados correspondientes a la dimensión "concepción del trabajo", permitieron identificar que el ítem "el trabajo colaborativo como método para desarrollar competencias sociales, argumentación, diálogo, capacidad de escucha, debate, respeto a opiniones discrepantes", obtuvo un valor promedio de 4,3 $\pm 0,8$; mientras que el ítem "la oportunidad para conocer mejor a los compañeros" obtuvo valor promedio de 4,0 \pm 0,9. La pregunta respecto a que "el trabajo grupal facilita la preparación para los exámenes" obtuvo un valor promedio de 3,7 $\pm 1,1$.

Por otro lado, en la dimensión "utilidad para su formación", las puntuaciones promedio fueron $\geq 4$. En las respuestas respecto a "llegar a un acuerdo ante las opiniones diferentes entre los estudiantes", el promedio de las puntuaciones fue 4,2 $\pm 0,8$; seguido de la "forma de entender los conocimientos e ideas de los compañeros", con un valor promedio $4,2 \pm 0,8$. Asimismo, el ítem "comprender la importancia del trabajo coordinado como elemento para el futuro docente", obtuvo 4,4 \pm 1,0 de puntuación promedio (Tabla 1).

Con respecto a los resultados de la dimensión "planificación del trabajo por parte del profesor", el ítem "la asistencia a clases prácticas permiten resolver las dudas que surgen en la elaboración del trabajo en grupo", obtuvo un puntuación promedio $4,2 \pm 0,8$; mientras que "la existencia de coordinación entre los trabajos de grupo solicitados en distintos cursos", obtuvo una puntuación promedio $3,5 \pm 1,1$.

En la dimensión "criterios para organizar grupos", los ítems "la incorporación y nombramiento de un coordinador de grupo" y "el grupo sea estable durante el desarrollo de curso", obtuvieron puntuaciones promedio 4,4 y 4,2 respectivamente. Asimismo, el ítem de "la organización de grupos se realiza por criterios de amistad", presentó una puntuación promedio de 3,4 \pm 1,2 (Tabla 1).

El número ideal de participantes para la conformación de grupos obtuvo un valor promedio de 5,1 $\pm 1,3$. Mientras tanto, en la dimensión "normas de funcionamiento", el ítem las "consecuencias que tendrían los participantes por no cumplir los compromisos asumidos", obtuvo un valor promedio $4,4 \pm 0,8$; mientras que se obtuvo 4,3 \pm 0,9 para la "definición de roles de los integrantes del grupo". Tanto las "normas establecidas por el estudiante" y las "establecidas por el profesor", obtuvieron un valor promedio de 3,7 , mientras que el valor promedio 2,0 correspondió a la pregunta de "no existir ninguna norma para el funcionamiento del grupo" (Tabla 1).

Los resultados correspondientes a la dimensión "funcionamiento interno de los grupos", para "realizar búsquedas de información en diferentes fuentes" y "consulta de documentación básica", obtuvieron un valor promedio de 4,3 $\pm 0,8 \mathrm{y}$ $4,2 \pm 0,8$, respectivamente, mientras que "la participación equitativa de todos los integrantes del grupo", obtuvo un valor promedio de $3,8 \pm 1,2$.

La última dimensión correspondiente a la "eficacia del trabajo grupal", en "labor del profesor para supervisar el trabajo del grupo" obtuvo un valor promedio de 4,5 \pm 0,8 y la "información previa que proporciona el profesor sobre los criterios de evaluación" tuvo un valor promedio de 4,4 $\pm 0,8$. Sin embargo, la "incorporación de la autoevaluación en la evaluación global del grupo", obtuvo un valor promedio de 3,9 \pm 0,9 (Tabla 1).

El análisis comparativo de resultados permitió observar que el grupo medio predomina en la dimensión "concepción del trabajo de grupo", con un resultado correspondiente a 40,5\% de estudiantes y $20,9 \%$ para el grupo alto. Asimismo, no existieron diferencias importantes entre los grupos en la dimensión "utilidad del trabajo de grupo para su formación". El grupo bajo se presentó en $41,9 \%$ de estudiantes en la dimensión "planificación del trabajo de los grupos por el profesor".

Por otra parte, en la dimensión "criterios para organizar grupos", el grupo medio se presentó en $37,8 \%$ de estudiantes, mientras que los grupos medio y alto obtuvieron resultados similares. En la dimensión "normas de funcionamiento de grupos", 37,2\% correspondió al grupo bajo y $28,4 \%$ para el grupo alto. Además, en la dimensión "funcionamiento interno", el grupo bajo se presentó en $43,2 \%$ y $27,7 \%$ para el grupo medio. En la dimensión "eficacia del trabajo grupal", el grupo bajo se presentó en 39,9\% de estudiantes y $32,4 \%$ para el grupo alto (Figura 1).

De acuerdo con las valoraciones totales establecidas, $22,3 \%$ de los estudiantes pertenecieron al grupo bajo y $23 \%$ al grupo medio; en ambos grupos con predominio de estudiantes del género fe- 
Tabla 1. Resultados de las valoraciones generales sobe el trabajo grupal por dimensiones del cuestionario de análisis del trabajo cooperativo en educación superior (ACOES), en estudiantes de medicina. Lima, 2019

\begin{tabular}{|c|c|c|c|c|c|c|c|}
\hline \multirow{2}{*}{ Ítems } & \multicolumn{2}{|c|}{ Hombres } & \multicolumn{2}{|c|}{ Mujeres } & \multicolumn{2}{|c|}{ Total } & \multirow{2}{*}{ Valor $\mathbf{p}$ * } \\
\hline & Promedio & DE & Promedio & DE & Promedio & DE & \\
\hline \multicolumn{8}{|l|}{ Concepción del trabajo de grupo } \\
\hline $\begin{array}{l}\text { Un buen método para desarrollar mis competencias sociales: } \\
\text { argumentación, dialogo, capacidad de escucha, debate, respeto } \\
\text { a opiniones discrepantes }\end{array}$ & 4,4 & 0,8 & 4,2 & 0,8 & 4,3 & 0,8 & 0,270 \\
\hline Una oportunidad para conocer mejor a mis compañeros/as & 4,0 & 0,9 & 4,0 & 0,9 & 4,0 & 0,9 & 0,330 \\
\hline Una forma de comprender mejor los conocimientos & 3,9 & 0,8 & 4,1 & 0,9 & 4,0 & 0,8 & 0,340 \\
\hline Una manera de compartir el volumen de trabajo total & 3,8 & 0,9 & 4,0 & 0,9 & 3,9 & 0,9 & 0,120 \\
\hline Una manera para facilitar la preparación para los exámenes & 3,7 & 1,0 & 3,8 & 1,1 & 3,7 & 1,1 & 0,380 \\
\hline \multicolumn{8}{|l|}{ Utilidad del trabajo en grupo para su formación } \\
\hline $\begin{array}{l}\text { Exponer y defender mis ideas y conocimientos ante otras } \\
\text { personas }\end{array}$ & 4,1 & 0,9 & 4,2 & 0,9 & 4,1 & 0,9 & 0,210 \\
\hline Sentirme parte activa de mi propio proceso de aprendizaje & 4,0 & 1,0 & 4,2 & 0,7 & 4,1 & 0,9 & 0,210 \\
\hline $\begin{array}{l}\text { Entender los conocimientos e ideas de los compañeros y } \\
\text { compañeras }\end{array}$ & 4,1 & 0,9 & 4,2 & 0,7 & 4,2 & 0,8 & 0,970 \\
\hline $\begin{array}{l}\text { Comprender la importancia del trabajo coordinado en mi futuro } \\
\text { profesional como docente }\end{array}$ & 4,0 & 0,9 & 4,1 & 1,0 & 4,0 & 1,0 & 0,710 \\
\hline Llegar a acuerdos ante opiniones diferentes & 4,1 & 0,9 & 4,2 & 0,8 & 4,2 & 0,8 & 0,420 \\
\hline Buscar información, investigar y aprender de forma autónoma & 4,1 & 1,0 & 4,3 & 0,8 & 4,2 & 0,9 & 0,330 \\
\hline \multicolumn{8}{|l|}{ Planificación del trabajo de los grupos por parte del profesor } \\
\hline $\begin{array}{l}\text { La cantidad de trabajos de grupo solicitados se adecuan a la } \\
\text { carga lectiva del curso }\end{array}$ & 4,1 & 0,8 & 3,9 & 0,9 & 4,0 & 0,9 & 0,272 \\
\hline $\begin{array}{l}\text { El nivel de dificultad de los trabajos de grupo es el adecuado } \\
\text { para nuestra formación }\end{array}$ & 4,1 & 0,9 & 4,0 & 0,8 & 4,0 & 0,8 & 0,359 \\
\hline $\begin{array}{l}\text { Existe coordinación entre los trabajos de grupo solicitados en } \\
\text { las distintas asignaturas }\end{array}$ & 3,5 & 1,1 & 3,4 & 1,1 & 3,5 & 1,1 & 0,332 \\
\hline $\begin{array}{l}\text { La asistencia a clases prácticas resuelve las dudas que me } \\
\text { surgen en la elaboración del trabajo en grupo }\end{array}$ & 4,2 & 0,8 & 4,2 & 0,8 & 4,2 & 0,8 & 0,756 \\
\hline \multicolumn{8}{|l|}{ Criterios para organizar grupos } \\
\hline Realizarla el alumnado aplicando criterios de amistad & 3,6 & 1,3 & 3,4 & 1,2 & 3,4 & 1,2 & 0,858 \\
\hline Realizarla el alumnado aplicando criterios académicos & 4,0 & 1,0 & 3,9 & 1,1 & 3,9 & 1,0 & 0,621 \\
\hline Realizarla el profesorado aplicando criterios académicos & 3,7 & 1,3 & 3,6 & 1,2 & 3,6 & 1,3 & 0,620 \\
\hline $\begin{array}{l}\text { Tener una composición diversa de los miembros del grupo } \\
\text { (edad, sexo, formación, experiencias,...) }\end{array}$ & 4,0 & 1,0 & 3,9 & 1,0 & 3,9 & 1,0 & 0,870 \\
\hline Ser estable a lo largo de la asignatura, cuatrimestre, curso,... & 4,0 & 0,9 & 4,3 & 0,8 & 4,2 & 0,8 & 0,063 \\
\hline $\begin{array}{l}\text { Modificarse para la realización de diferentes actividades en una } \\
\text { misma asignatura }\end{array}$ & 3,6 & 1,1 & 3,4 & 1,2 & 3,5 & 1,1 & 0,194 \\
\hline $\begin{array}{l}\text { Incorporar el nombramiento de un coordinador o coordinadora } \\
\text { del grupo }\end{array}$ & 4,3 & 1,0 & 4,4 & 0,8 & 4,4 & 0,9 & 0,595 \\
\hline \multicolumn{8}{|l|}{ Normas de funcionamiento de los grupos } \\
\hline No debe existir ninguna norma & 2,1 & 1,4 & 2,0 & 1,3 & 2,0 & 1,3 & 0,769 \\
\hline Deben existir normas, pero establecidas por el alumnado & 3,6 & 1,0 & 3,8 & 1,0 & 3,7 & 1,0 & 0,138 \\
\hline Deben existir normas, pero establecidas por el profesorado & 3,6 & 1,2 & 3,8 & 1,0 & 3,7 & 1,1 & 0,460 \\
\hline Deben ser negociadas entre el profesorado y el alumnado & 3,9 & 1,2 & 4,2 & 1,0 & 4,1 & 1,1 & 0,067 \\
\hline $\begin{array}{l}\text { Deben estar recogidas en un documento donde se concreten } \\
\text { las responsabilidades que asume el grupo }\end{array}$ & 3,9 & 1,1 & 3,8 & 1,1 & 3,8 & 1,1 & 0,430 \\
\hline $\begin{array}{l}\text { Deben definir los roles que van a desempeñar cada una de las } \\
\text { personas que constituyen el grupo }\end{array}$ & 4,2 & 1,0 & 4,5 & 0,7 & 4,3 & 0,9 & 0,147 \\
\hline $\begin{array}{l}\text { Deben incluir las consecuencias que tendrían para los } \\
\text { participantes no cumplir los compromisos asumidos }\end{array}$ & 4,3 & 0,9 & 4,4 & 0,8 & 4,4 & 0,8 & 0,632 \\
\hline Deben concretar el horario y lugar de las reuniones & 4,2 & 1,0 & 4,4 & 0,8 & 4,3 & 0,9 & 0,547 \\
\hline Deben incluir la obligatoriedad de asistir a las reuniones & 4,1 & 0,9 & 4,3 & 0,9 & 4,2 & 0,9 & 0,215 \\
\hline
\end{tabular}

* Prueba U de Mann-Whitney. (Continuá en la página 461) 
Tabla 1. Resultados de las valoraciones generales sobe el trabajo grupal por dimensiones del cuestionario de análisis del trabajo cooperativo en educación superior (ACOES), en estudiantes de medicina. Lima, 2019 (Viene de la página 460)

\begin{tabular}{|c|c|c|c|c|c|c|c|}
\hline \multirow{2}{*}{ Ítems } & \multicolumn{2}{|c|}{ Hombres } & \multicolumn{2}{|c|}{ Mujeres } & \multicolumn{2}{|c|}{ Total } & \multirow{2}{*}{$\begin{array}{l}\text { Valor } \\
\text { p* }\end{array}$} \\
\hline & Promedio & DE & Promedio & DE & Promedio & $\mathrm{DE}$ & \\
\hline \multicolumn{8}{|l|}{ Funcionamiento interno de los grupos } \\
\hline $\begin{array}{l}\text { Nos reunimos al inicio para planificar los diferentes pasos que tenemos que } \\
\text { realizar }\end{array}$ & 3,9 & 1,0 & 4,1 & 1,1 & 4 & 1,0 & 0,241 \\
\hline Consultamos la documentación básica aportada por el profesor & 4,1 & 1,0 & 4,2 & 0,7 & 4,2 & 0,8 & 0,760 \\
\hline $\begin{array}{l}\text { Realizamos búsqueda de información en diferentes fuentes (internet, biblioteca, } \\
\text {...) }\end{array}$ & 4,2 & 0,9 & 4,4 & 0,8 & 4,3 & 0,8 & 0,475 \\
\hline $\begin{array}{l}\text { Tomamos decisiones, de forma consensuada, para garantizar la coherencia global } \\
\text { del trabajo de grupo }\end{array}$ & 3,9 & 1,2 & 4,3 & 0,8 & 4,1 & 1,0 & 0,182 \\
\hline $\begin{array}{l}\text { Durante la realización del trabajo hacemos “puestas en común" para que todo } \\
\text { el grupo conozca lo que los demás están haciendo y tengamos buena idea de la } \\
\text { marcha de la actividad }\end{array}$ & 4,1 & 0,9 & 4,2 & 0,9 & 4,1 & 0,9 & 0,291 \\
\hline Participamos equitativamente todos los componentes del grupo & 3,8 & 1,2 & 3,9 & 1,2 & 3,8 & 1,2 & 0,536 \\
\hline Lo evaluamos y hacemos propuestas de mejora & 4,1 & 1,1 & 4,1 & 1,0 & 4,1 & 1,0 & 0,995 \\
\hline \multicolumn{8}{|l|}{ Eficacia del trabajo grupal } \\
\hline El profesorado facilita unas pautas claras de las actividades grupales a desarrollar & 4,4 & 0,9 & 4,4 & 0,8 & 4,4 & 0,8 & 0,700 \\
\hline $\begin{array}{l}\text { Las actividades planteadas por el profesorado requieren que haya análisis, debate, } \\
\text { reflexión y crítica }\end{array}$ & 4,1 & 0,9 & 4,3 & 0,7 & 4,2 & 0,8 & 0,102 \\
\hline El profesorado supervisa el trabajo del grupo & 4,4 & 0,8 & 4,5 & 0,7 & 4,5 & 0,8 & 0,913 \\
\hline El profesorado controla la asistencia regular a clase & 4,3 & 0,9 & 4,3 & 0,9 & 4,3 & 0,9 & 0,856 \\
\hline Los trabajos se valoran adecuadamente en la calificación global de la asignatura & 4,1 & 0,9 & 4,2 & 0,9 & 4,1 & 0,9 & 0,776 \\
\hline $\begin{array}{l}\text { El profesorado nos informa previamente sobre los criterios de evaluación de la } \\
\text { actividad de grupo }\end{array}$ & 4,4 & 0,8 & 4,5 & 0,8 & 4,4 & 0,8 & 0,320 \\
\hline $\begin{array}{l}\text { El profesorado evalúa los diferentes niveles de participación de cada uno de los } \\
\text { miembros del grupo }\end{array}$ & 4,2 & 1,0 & 4,3 & 0,9 & 4,2 & 0,9 & 0,497 \\
\hline Se incorpora la autoevaluación de cada alumno/a en la evaluación global del grupo & 3,9 & 0,9 & 4 & 1,0 & 3,9 & 0,9 & 0,553 \\
\hline Nos evaluamos los miembros del grupo unos a otros & 3,7 & 1,1 & 3,8 & 1,0 & 3,7 & 1,1 & 0,765 \\
\hline $\begin{array}{l}\text { El profesorado le asigna al trabajo de grupo un peso importante en la calificación } \\
\text { final de la asignatura }\end{array}$ & 4,1 & 1,0 & 4,1 & 0,9 & 4,1 & 0,9 & 0,818 \\
\hline
\end{tabular}

* Prueba U de Mann-Whitney.

menino. De otro lado, 54,7\% perteneció al grupo alto, con una mayor presencia del género masculino. El análisis bivaria- do entre el género de los estudiantes y los grupos obtuvieron un valor $p=0,115$, evidenciando que no existe relación en- tre ambas variables estudiadas. Con respecto a los resultados correspondiente al rendimiento académico, los estudiantes

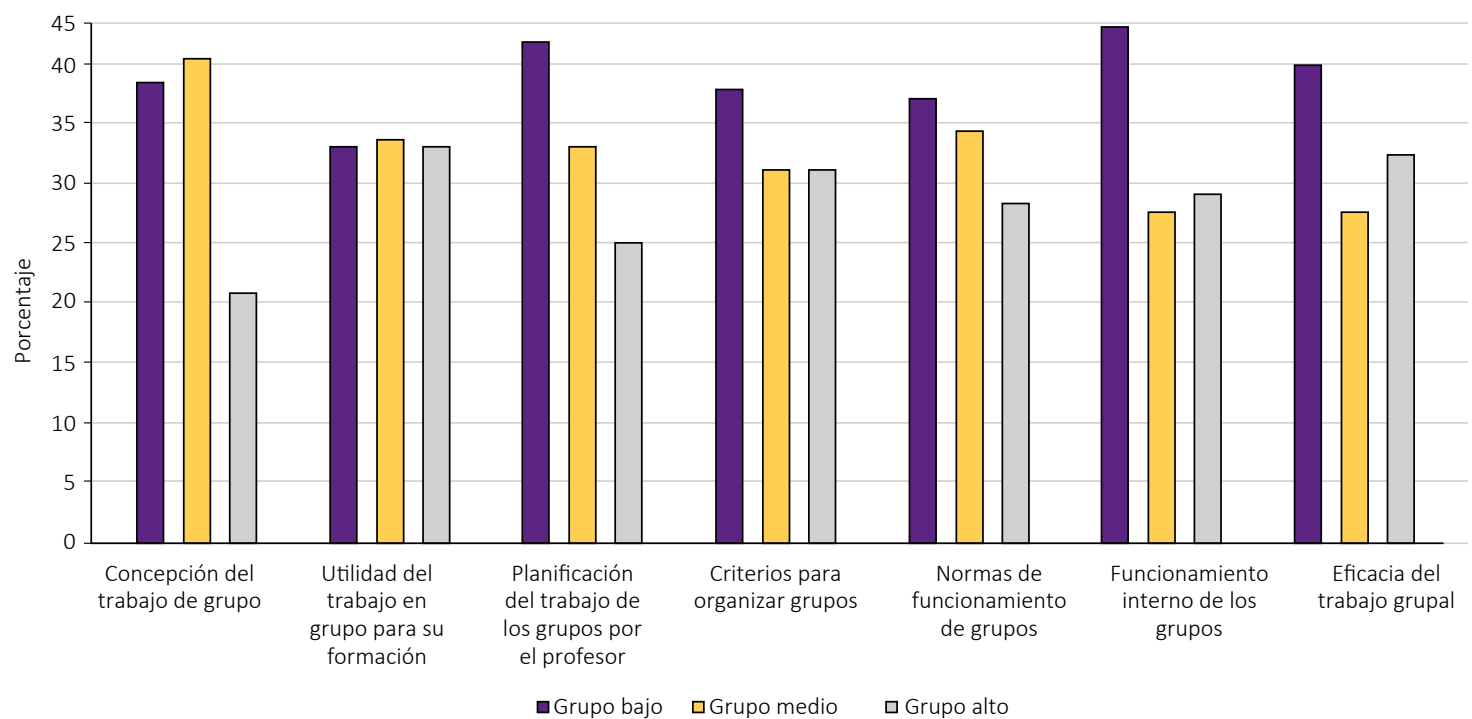

Figura 1. Resultados de la valoración del trabajo colaborativo distribuido por grupos, en estudiantes de medicina. Lima, 2019. 
de género femenino obtuvieron una nota promedio final de $12,9 \pm 1,3$, mientras que $12,5 \pm 1,3$ para el masculino. El promedio global correspondió a $12,7 \pm 1,4$ los resultados no presentaron diferencias significativas.

El análisis bivariado entre el rendimiento académico en función al promedio de notas y trabajo colaborativo permitió observar que el rendimiento académico de los estudiantes del grupo bajo y medio son similares $(p>0,05)$, mientras que el rendimiento académico de los estudiantes de género femenino ubicados en el grupo alto es significativamente mayor en comparación con los estudiantes de género masculino $(p=0,049)$ (Tabla 2).

\section{DISCUSIÓN}

Con el desarrollo de la globalización, el interés en el aprendizaje colaborativo activo ha permitido que los estudiantes se vuelvan más capaces de comunicarse con sus compañeros para resolver problemas u organizar diversas actividades de manera colaborativa ${ }^{(9)}$. Algunos investigadores han informado que el aprendizaje colaborativo tiene un impacto positivo en los estudiantes debido a que son capaces de realizar actividades, como discutir sus tareas con sus compañeros y recibir comentarios antes de presentar un trabajo final ${ }^{(10,11)}$. En términos de conocimiento, Janssen y col. plantearon que el aprendizaje colaborativo es mucho mas importante cuando los estudiantes estan equipados con capacidad cognitiva ${ }^{(10)}$.

Actualmente las brechas respecto al género de los estudiantes de medicina se han acortado y, en algunos casos, existe un mayor número de estudiantes de género femenino, como lo ratifica una investigación realizada en siete facultades de medicina del Perú ${ }^{(12)}$. Sin embargo, otras investigaciones han reportado una presencia de 59,4\% de estudiantes de género masculino, como los realizados en una Universidad Pública de Pereira en Colombia ${ }^{(13)}$

El trabajo colaborativo se ha convertido en una forma eficaz para el desarrollo de habilidades de pensamiento crítico e interacción social de los estudiantes, como consecuencia de desarrollar trabajos con la ayuda de sus compañeros ${ }^{(14)}$. Asimismo, las habilidades comunicativas siguen siendo elementos mejor valoradas por los estudiantes, dentro de los cuales se podrían mencionar, escuchar al compañero, respeto a opiniones y la comunicación en forma asertiva ${ }^{(15)}$, las mismas que son coincidentes con los hallazgos descritos en relación con el desarrollo de competencias sociales, capacidad de escucha y respeto a opiniones discrepantes.

Es importante señalar que el trabajo colaborativo no solo es útil para el desarrollo de contenidos y la adquisición de conocimientos teóricos, sino que debe ser una propuesta educativa o forma de trabajo que fomente el desarrollo de competencias profesionales que serán necesarias en un futuro mediato, cuando los estudiantes se conviertan en profesionales y asuman responsabilidades laborales, como lo describe una investigación realizada en la Universidad de Zaragoza ${ }^{(16)}$. En esa misma orientación, los estudiantes manifestaron respuestas similares respecto a que el trabajo colaborativo es importante para su formación profesional, ya que actualmente se considera que la adquisición de competencias básicas es indispensable para el aprendizaje de una profesión y estas deben de ser adquiridas desde los inicios de la educación universitaria ${ }^{(17)}$.

La coordinación de los grupos presen- ta algunas limitaciones, debido que no existe una adecuada coordinación entre los profesores de asignaturas, lo que ocasiona que cada curso conforme grupos distintos, obligando a los integrantes a asistir a las sesiones para tratar de aclarar diversas dudas como, formulación de una hipótesis, identificar una variable, calcular una muestra y elegir el tipo de estudio. Por ello se considera necesario el desarrollo de guías de actividades prácticas para facilitar el trabajo colaborativo, ya que permite ayudar a planificar de mejor forma el trabajo en los diferentes niveles de aprendizaje ${ }^{(16,18)}$.

La conformación de grupos aún se desarrolla tomando diversos criterios: normativas de la universidad, normas propias de los profesores y criterios de amistad de los estudiantes, que son aplicables para un curso específico. Estos hallazgos están respaldados por Jaén Martínez, donde se enfatiza que la estructura organizativa de los grupos se realiza tomando en cuenta la organización previa de los grupos creados ${ }^{(19)}$. Uno de los criterios que presenta diversas opiniones, corresponde al número de integrantes de cada grupo. Algunos recomiendan que los grupos deben estar conformados por tres estudiantes, ya que consideran que a mayor cantidad de integrantes la responsabilidad se concentrará en uno o dos miembros del grupo y la intención es que todos los integrantes colaboren en forma equitativa ${ }^{(20)}$. Contrariamente a los resultados, los estudiantes manifestaron que los grupos deben tener cinco integrantes.

Según Leris, existe unanimidad para implementar normas que regulen el funcionamiento de los grupos; sin embargo, existen diferencias para designar al responsable que establezca las normas. Algunos manifiestan que las normas deberían estar a cargo del profesor o deberían estar establecidas por estudiantes y

Tabla 2. Análisis comparativo entre rendimiento académico y el trabajo colaborativo según grupos, en estudiantes de medicina ( $n=166)$. Lima, 2019.

\begin{tabular}{lccccccc} 
& \multicolumn{7}{c}{ Promedio de notas } \\
\cline { 2 - 7 } Grupos & \multicolumn{2}{c}{ Hombres } & \multicolumn{2}{c}{ Mujeres } & & Total & Valor $\mathbf{p}$ \\
\cline { 2 - 8 } & Promedio & DE & Promedio & DE & Promedio & DE \\
\hline Grupo bajo & 11,9 & 2,2 & 12,9 & 1,2 & 12,6 & 1,6 & 0,206 \\
\hline Grupo medio & 12,7 & 1,2 & 13,1 & 0,9 & 12,9 & 1,1 & 0,201 \\
\hline Grupo alto & 12,6 & 1,1 & 12,9 & 1,6 & 12,7 & 1,3 & 0,049 \\
\hline
\end{tabular}


un menor grupo, prefieren que las normas deberían ser establecidas entre el profesor y los estudiantes, ya que los grupos mixtos fomentan el desarrollo de habilidades de pensamiento ${ }^{(14,16)}$. Lo anteriormente descrito presenta coincidencias respecto a que las normas deben ser establecidas por el estudiante y el profesor, ya que aseguran que cada integrante asume responsabilidades y realiza un seguimiento de los resultados que va logrando.

Una dificultad que presentan los grupos en su funcionamiento es la participación no equitativa de los integrantes. Si bien es cierto que los resultados mostraron un valor promedio considerado regular, lo cual podría ocasionar que un solo miembro del grupo asuma toda la responsabilidad. Cabe señalar que la metodología del aprendizaje colaborativo permite que los estudiantes asuman nuevos retos desarrollando capacidades de comunicación e investigación que son difíciles de lograr en sesiones educativas tradicionales. Por ello, la asignación de roles específicos a cada integrante del grupo permite un trabajo equitativo y fomenta la capacidad de liderazgo, mediante una responsabilidad rotativa y compartida ${ }^{(21)}$.

La supervisión del trabajo es responsabilidad del profesor, el mismo que puede observar diversos comportamientos del trabajo grupal, solución de problemas que se originen dentro del proceso y la evaluación de logros, como lo sostiene coincidentemente Mora-Vicaroli ${ }^{(20)}$. Otra de las funciones claves del profesor se centra en la información previa de los criterios de evaluación. Los resultados presentaron una orientación muy favorable y esta debe ser desarrollada en forma objetiva e imparcial, incorporando diferentes sistemas de evaluación en forma continua que, si son bien diseñados, no sería necesario aplicar un examen final, como lo recomienda un estudio realizado con estudiantes de enfermería ${ }^{(22)}$. Asimismo, Mora-Vicaroli menciona que los estudiantes manifestaron que las instrucciones proporcionadas por el profesor para el desarrollo de evaluaciones eran entre muy buenas y excelentes (20), las cuales podrían considerarse similares, ya que se encuentran dentro de la valoración "favorables".
Asimismo, los resultados reflejan un acuerdo para considerar a la autoevaluación como parte de la evaluación global. Cabe señalar que la individualización de las evaluaciones por niveles de participación de cada integrante de un grupo puede tener aceptación ${ }^{(16)}$. Sin embargo, las autoevaluaciones pueden presentar algunas brechas, ya que ocasionalmente las evaluaciones teóricas no coinciden con las prácticas y se hace necesario implementar sesiones de diálogo abierto entre estudiantes y profesor ${ }^{(18)}$.

Con respecto al rendimiento académico, el promedio final de notas no superó la calificación de 14 según escala vigesimal, la cual se considera regular. Estas no presentaron relación con las valoraciones, solo se evidencia un mejor rendimiento académico en estudiantes de género femenino del grupo alto. Contrariamente, los resultados de una investigación realizada en una Universidad de Panamá reportaron un incremento de las calificaciones en estudiantes que desarrollaron actividades interactivas ${ }^{(23)}$. Otra investigación realizada en Hong Kong y Malasia, enfatizó la importancia del aprendizaje colaborativo como elemento de motivación de los estudiantes, mientras la interactividad y el compromiso tienen una influencia positiva en el rendimiento académico ${ }^{(24,25)}$. Asimismo, se la logrado demostrar que los estudiantes que trabajan colaborativamente, son obligados a documentarse, investigar, reflexionar y preparar sus exposiciones y que estos tienen mejor rendimiento académico, lo que permite que más del $90 \%$ de los estudiantes obtenga calificaciones sobresalientes y notables ${ }^{(18,22)}$.

La presente investigación tiene algunas limitaciones: datos recolectados mediante una corte transversal, no pueden generalizarse los resultados a otros contextos, y ausencia de estandarización de procesos de enseñanza y aprendizaje. Asimismo, se utilizó una muestra no probabilística lo cual limita la inferencia de los resultados de la investigación

Finalmente, se concluye que el trabajo colaborativo es una metodología dinámica, que fomenta la participación activa de estudiantes. La valoración como grupo bajo predomina en las dimensiones planificación del trabajo de grupos por el profesor, criterios para organizar grupos, normas de funcionamiento, funcionamiento interno y eficacia de grupos. El grupo alto alcanzó una valoración de $32,4 \%$ en la dimensión eficacia del trabajo grupal, la valoración global fue de $54,7 \%$ de estudiantes para el grupo alto y 12,7 como promedio global de calificaciones.

\section{REFERENCIAS BIBLIOGRÁFICAS}

1. Pico L, Rodríguez C. Trabajos colaborativos. Serie estrategias en el aula para el modelo 1 a 1. Buenos Aires: Educ.ar SE; 2011. 56p.

2. Martinez-Martin ML, Canalejas-Pérez C, ParroMoreno Al, Cid-Galán ML, Garcia- González A, Martín-Salinas C. Estrategia colaborativa para la integración de competencias en la formación de grado de enfermeria. Educación Médica. 2018; 19(4): 201-7. DOI: 10.1016/j.edumed.2017.03.006

3. Cenich G, Santos G. Propuesta de aprendizaje basado en proyecto y trabajo colaborativo: experiencia de un curso en línea. REDIE. Revista Electrónica de Investigación Educativa. 2005; 7(2): 1-18.

4. Jarauta B. El aprendizaje colaborativo en la universidad: referentes y práctica. REDU. Revista de Docencia Universitaria. 2014; 12(4): 281-302. DOI: 10.4995/redu.2014.5624

5. Garcia M, Gonzáles I, Mérida R. Validación del cuestionario de evaluación ACOES. Análisis del trabajo cooperativo en educación superior. Revista de Investigación Educativa. 2012; 30(1): 87-109. DOI: 10.6018/rie.30.1.114091

6. Delgado M, Fasce E, Pérez C, Rivera N, Salazar P, Riquelme $\mathrm{C}$, et al. Trabajo en equipo y rendimiento académico en un curso de kinesiología empleando aprendizaje basado en equipos. Investigación en Educación Médica. 2017; 6(22): 80-87. DOI: 10.1016/j.riem.2016.05.006

7. Matzumura-Kasano JP, Gutiérrez-Crespo H, Pastor-Garcia C, Zamudio-Eslava- L, Ruiz-Arias Raúl. Metodología activa y estilos de aprendizaje en el proceso de enseñanza en el curso de metodología de la investigación de una facultad de ciencias de la salud. An. Fac. med. 2018; 79(4): 293-300. DOI: 10.15381/anales.v79i4.15632.

8. Matzumura-Kasano JP, Gutiérrez-Crespo $\mathrm{H}$, Zamudio-Eslava L, Zavala-Gonzales JC. Aprendizaje invertido para la mejora y logro de metas de aprendizaje en el Curso de Metodologia de la Investigación en estudiantes de universidad. Revista Electrónica Educare. 2018; 22(3): 177-97. DOI: 10.15359/ree.22-3.9.

9. Anderson J, Rainie L. Millennias will benefit and suffer due to their hyper connected lives. Pex Research Center. Internet \& Technology [Internet]. 2012 [Citado 28 oct 2019]. Disponible en: https://www.pewresearch.org/internet/2012/02/29/ millennials-will-benefit-and-suffer-due-to-theirhyperconnected-lives/

10. Janssen J, Kirschner F, Erkens G, Kirschner PA, Paas F. Making the black box of collaborative learning transparent: combining process-oriented and cognitive load approaches. Educ Psychol 
Rev. 2010; 22(2):139-54. DOI: 10.1007/s10648010-9131-x

11. Zhu C. Student satisfaction, performance and knowledge construction in online collaborative learning. J Educ Technol Soc. 2012; 15(1): 127-36.

12. Mejia $C R$, Valladares-Garrido $M$, Talledo-Ulfe $L$, Sánchez-Arteaga K, Rojas C. Ruiz-Arimuya J, et al. Sindrome de Burnout y factores asociados en estudiantes de medicina. Estudio multicéntrico en siete facultades de medicina peruanas. Rev. chil. neuro-psiquiatr. 2016; 54(3): 207-14. DOI: 10.4067/ S0717-92272016000300005

13. Machado-Duque ME, Echeverri J, Machado-Alba J. Somnolencia diurna excesiva, mala calidad de sueño y bajo rendimiento académico en estudiantes de Medicina. Rev Colomb Psiquiat. 2015; 44(3): 137-42. DOI: 10.1016/j.rcp.2015.04.002

14. Kulikovskikh IM, Prokhorov SA, Suchkova SA. Promoting collaborative learning through regulation of guessing in clickers. Computers in $\mathrm{Hu}$ man Behavior. 2017; 75: 81-91. DOI: 10.1016/j. chd.2017.05.001

15. León del Barco B, Felipe E, Iglesias D y Marugán $M$. Determinantes en la eficacia del aprendizaje cooperativo. Una experiencia en el EEES. Revista de Investigación Educativa. 2014; 32(2): 411-24.
DOI: 10.6018/rie.32.2.172721

16. Leris D, Vea F, Velamazán MA, Florentín P. Objetivos básicos del aprendizaje de Trabajo en Equipo en la Universidad. En: IV Congreso Internacional sobre Aprendizaje, Innovación y Competitividad; 2017 octubre 4-6; Zaragoza, España: 2017. 50914. DOI: 10.26754/CINAIC.2017.000001_107

17. Véliz PL, Blanco A, Ortiz M, Díaz L, Blanco O. Resultados de trabajo del Grupo para el Estudio de Competencias en Salud. Educación Médica Superior. 2018; 32(1): 106-117.

18. Navarro I, González C, López B, Botella P. Aprendizaje de contenidos académicos y desarrollo de competencias profesionales mediante prácticas didácticas centradas en el trabajo cooperativo y relaciones multidisciplinarias. Revista de Investigación Educativa. 2015; 33(1): 99-117. DOI: 10.6018/ rie.33.1.183971

19. Jaén A, Sirignano F. El aprendizaje cooperativo como estrategia didáctica para la adquisición de competencias en el EEES. Propuesta y reflexión sobre una experiencia. Revista Educativa Digital Hekademos. 2016; 19(8): 7-19

20. Mora-Vicarioli F y Hooper-Simpson C. Trabajo colaborativo en ambientes virtuales de aprendizaje: Algunas reflexiones y perspectivas estudiantiles.
Revista Electrónica Educare. 2016; 20(2): 1-26 DOI: 10.15359/ree.20-2.19

21. Medina-Gonzáles S. Aprendizaje colaborativo. Educación: Revista de la Facultad de Ciencias de la Educación. 2017; 23: 101-5. DOI: 10.33539/ educacion.2017.n23.1175

22. Martín-Salinas C, Cid-Galán L. Experiencia de apendizaje cooperativo en una asignatura optativa del Grado en Enfermería. Educación Médica. 2018; 19(5): 288-93. DOI: 10.1016/j.edumed.2017.10.035

23. Dorati Y, De Crespo M, Cantú F. El aprendizaje cooperativo aplicado a las matemáticas y sus efectos en el rendimiento académico. Prisma Tecnológico. 2016; 7(1): 26-9.

24. Chan S, Wan J, Ko S. Interactivity, active collaborative learning, and learning performance: The moderating role of perceived fun by using personal response systems. The International Journal of Management Education. 2019; 17(1): 94-102. DOI: 10.1016/j.ijme.2018.12.004

25. Al-Rahmi WM, Zeki AM. A model of using social media for collaborative learning to enhance learners performance on learning. Journal of King Saud University - Computer and Information Sciences. 2017; 29(4): 526-35. DOI: 10.1016/j.jksuci.2016.09.002 